\title{
Allelic analysis of low molecular weight glutenin subunits using 2-DGE in Korean wheat cultivars
}

\author{
Jong-Yeol Lee*1), You-Ran Jang'), Hye-Rang Beom ${ }^{1)}$, Susan B. Altenbach ${ }^{2)}$, Sun-Hyung Lim ${ }^{1)}$ \\ and Choung Keun Lee ${ }^{1)}$ \\ 1) National Institute of Agricultural Science, RDA, Jeonju, 54874, Korea \\ 2) USDA-ARS, Western Regional Research Center, 800 Buchanan Street, Albany, CA 94710, USA
}

\begin{abstract}
Two-dimensional gel electrophoresis (2-DGE) was used as a complement to SDS-PAGE to determine the allelic compositions of LMW-GS in 32 Korean wheat cultivars. Protein patterns generated by 2-DGE from each cultivar were compared to patterns from standard wheat cultivars for each allele. At the Glu-A3 locus, thirteen $c$, twelve $d$, three $e$ (null), two g and two new alleles were identified. At the Glu-B3 locus, one $b$, nineteen $d$, four $h$, one $i$ and five $a d$ alleles were identified. At the Glu-D3 locus, twenty-three $a$, four $b$, four $c$ and one $l$ alleles were identified. When compared to results obtained previously using SDS-PAGE, there were discrepancies in the allelic designations of 10 of 32 cultivars (31\%). While SDS-PAGE is a rapid and relatively simple method for assessing LMW-GS composition, the similar mobilities of the proteins makes it difficult to discriminate certain alleles. 2-DGE is a more complicated technique, but provides a more accurate picture of the complement of the LMW-GS in a given cultivar. In addition to providing essential information for wheat breeders, the 2-DGE reference maps generated in this study will make it possible to study the contributions of individual LMW-GS to flour quality.
\end{abstract}

Key Words: 2-DGE, allelic analysis, flour quality, LMW-GS, Triticum aestivum L.

\section{Introduction}

The complement of gluten proteins accumulated during wheat grain development determines the viscoelastic properties that make it possible to produce a wide range of breads, noodles and baked goods from wheat flour. The gluten proteins consist of two complex groups of proteins, referred to as gliadins and glutenins. The gliadins are present in the flour as monomers and include three protein types on the basis of sequence, $\alpha / \beta-, \gamma$ - and $\omega$-gliadins. In comparison, the glutenins are large polymers consisting of two protein types that are linked together by disulfide bonds, high molecular weight glutenin subunits (HMW-GS) of about 66 to $88 \mathrm{kDa}$ and low molecular weight glutenin subunits (LMW-GS) of about 30 to $40 \mathrm{kDa}$. Most bread wheat cultivars express three to five different HMW-GS and 20 to 30 different LMW-GS. The HMW-GS are encoded by genes at the Glu-A1, Glu-B1, and Glu-D1 loci on the long arms of chromosomes 1A, 1B, and 1D, respectively (Payne et al.

Communicated by Donghe Xu

Received June 27, 2016. Accepted April 16, 2017.

First Published Online in J-STAGE on August 11, 2017.

*Corresponding author (e-mail: jy0820@korea.kr)
1980) while the LMW-GS are encoded at the Glu-A3, Glu-B3, and Glu-D3 loci on the short arms of the same chromosomes (Gupta and Shepherd 1990). Considerable allelic variation exists at both the Glu-1 and the Glu-3 loci and has been associated with differences in the functional properties of wheat flour. As a result, knowledge about the allelic compositions of parental lines is critical for wheat breeding programs aimed at improving flour quality.

The HMW-GS can be separated from other gluten proteins and from each other by SDS-PAGE and their allelic variation often is assessed by this method. A number of methods have been used to characterize the more complex LMW-GS alleles, including SDS-PAGE, two-dimensional gel electrophoresis (2-DGE), polymerase chain reaction (PCR), and matrix-assisted laser desorption/ionization time of flight mass spectrometry (MALDI-TOF-MS). As summarized by Liu et al. (2010), each method has certain limitations. Although SDS-PAGE is a widely used technique, a high level of expertise is required to interpret results because of the complex banding patterns of the LMW-GS. The LMW-GS also overlap considerably with gliadins in SDSPAGE. Additionally, some alleles are not reliably distinguished by this method. 2-DGE in which proteins are separated according to isoelectric point (pI) and molecular mass 
(MW) has been used to distinguish many but not all LMWGS alleles and the increased resolution of 2-DGE has resulted in the identification of new alleles not observed by SDSPAGE (Ikeda et al. 2006, Lee et al. 2016, Liu et al. 2010, Luo et al. 2015, Zhang et al. 2012, Zhen et al. 2014). PCR is a much simpler technique and primers specific for many alleles at the Glu-A3 and Glu-B3 loci have been developed and are currently used in breeding programs (Wang et al. 2009, 2010, Zhang et al. 2004, 2012, Zhao et al. 2006). However, primers are not available for all LMW-GS alleles, particularly those identified recently. Additionally, PCR has not proved to be useful for discriminating alleles at the Glu-D3 locus because of the few differences in DNA sequences. MALDI-TOF-MS is better at distinguishing alleles at the Glu-D3 locus but falls somewhat short in discriminating certain alleles at the $G l u-A 3$ and the $G l u-B 3$ loci. Additionally, this approach requires expensive instrumentation that is not readily available to many breeding programs (Liu et al. 2010).

In Korea, 38 wheat varieties have been developed since the 1970s. Early wheat breeding programs focused on developing high yielding wheat as well as early maturing varieties that were adapted to the Korean rainy season. Around the year 2000, Korean wheat breeders began to consider end-use quality in their breeding programs and focused on the Glu-1 loci because of well-documented effects on quality (Belton 1999, Payne 1987, Shewry et al. 1992). More recently, reports on the importance of LMW-GS in flour quality (Gupta and Shepherd 1990, Gupta and MacRitchie 1994, Maruyama-Funatsuki et al. 2004, Zhang et al. 2012) made it clear that LMW-GS should also be considered as breeding targets. Thus, it became essential to survey the LMW-GS alleles in Korean germplasm. This was first accomplished using SDS-PAGE (Park et al. 2011, Shin et al. 2012). In this study, we use 2-DGE to confirm the allelic compositions of LMW-GS in 32 Korean wheat cultivars and to generate proteomic maps that will be used in future studies to link specific LMW-GS to quality.

\section{Materials and Methods}

\section{Plant materials}

Grain from 32 Korean hexaploid wheat (Triticum aestivum L.) cultivars was harvested in 2012 and 2013 by RDA National Institute of Crop Sciences, Jeonju, Korea. Standard wheat cultivars used for the identification of Glu-3 alleles were kindly provided by National Plant Germplasm System of the USDA-ARS, USA. These included the cultivars Cheyenne, Chinese Spring, Gabo, Glenlea, Insignia, Marquis, Nanbu-Komugi, Norin 61, Orca, Opata and Suneca (Table 1). Grain was crushed with a cyclone sample mill (Udy Corporation, Fort Collins, USA).

\section{Glutenin extraction}

The glutenin was extracted using the procedure of Singh et al. (1991). Five hundred $\mathrm{mg}$ of flour was extracted with $25 \mathrm{ml}$ of $50 \%(\mathrm{v} / \mathrm{v})$ propanol for $30 \mathrm{~min}$ at $65^{\circ} \mathrm{C}$. After cen-
Table 1. Standard wheat cultivars used to distinguish alleles at the Glu-A3, Glu-B3 and Glu-D3 loci by 2-DGE

\begin{tabular}{|c|c|}
\hline Alleles & Standard cultivars \\
\hline Glu-A3a & Chinese Spring \\
\hline$G l u-A 3 b$ & Gabo \\
\hline$G l u-A 3 c$ & Cheyenne \\
\hline Glu-A3d & Orca \\
\hline Glu-A3e (null) & Marquis \\
\hline Glu-A3f & Insignia \\
\hline Glu-A3g & Glenlea \\
\hline Glu-B3a & Chinese Spring \\
\hline$G l u-B 3 b$ & Gabo \\
\hline$G l u-B 3 a b$ & Nanbu-Komugi \\
\hline$G l u-B 3 c$ & Insignia \\
\hline$G l u-B 3 d$ & Orca \\
\hline$G l u-B 3 g$ & Glenlea \\
\hline Glu-B3h & Suneca \\
\hline$G l u-B 3 i$ & Norin61 \\
\hline Glu-B3ad & Opata \\
\hline Glu-D3a & Chinese Spring \\
\hline$G l u-D 3 b$ & Gabo \\
\hline$G l u-D 3 c$ & Insignia \\
\hline
\end{tabular}

trifugation at $10,000 \mathrm{~g}$ for $5 \mathrm{~min}$, the supernatant fraction containing gliadin was removed. This extraction was repeated on the pellet two times. The precipitate was then extracted with $2.5 \mathrm{ml} 50 \%$ (v/v) propanol, $0.08 \mathrm{M}$ Tris- $\mathrm{HCl} \mathrm{pH} 8.0$ containing $1 \%(\mathrm{w} / \mathrm{v})$ dithiothreitol (DTT) at $65^{\circ} \mathrm{C}$ for $30 \mathrm{~min}$. After centrifugation at 10,000 $\mathrm{g}$ for $5 \mathrm{~min}, 2.5 \mathrm{ml}$ of the same buffer containing 1.4\% 4-vinylpyridine $(\mathrm{v} / \mathrm{v})$ instead of $1 \%$ DTT was added for alkylation at $65^{\circ} \mathrm{C}$ for $15 \mathrm{~min}$. After centrifugation at $10,000 \mathrm{~g}$ for $2 \mathrm{~min}$, the supernatant was moved to a new $1.5 \mathrm{ml}$ tube and stored at $-20^{\circ} \mathrm{C}$ overnight.

\section{Two-dimensional gel electrophoresis}

For two-dimensional gel electrophoresis (2-DGE), the extracted glutenin fractions were precipitated using $15 \%$ (v/v) TCA/acetone at $-20^{\circ} \mathrm{C}$ for 3 hours and the resulting glutenin pellets were mixed completely in $70 \mu$ of rehydration buffer (7 M urea, $2 \mathrm{M}$ thiourea, $2 \%$ CHAPS, $0.5 \%$ IPG buffer) (GE Healthcare Life Sciences, USA) containing $12.8 \mu 11 \mathrm{M}$ DTT. Protein was quantified by the method of Bradford (1976). Fifty $\mu \mathrm{g}$ of glutenin in $350 \mu$ rehydration buffer containing $12.8 \mu \mathrm{l} 1 \mathrm{M}$ DTT was loaded onto an $18 \mathrm{~cm}$ IPG strip (pI 6-11 or 3-11) (GE Heathcare Life Sciences, USA) and rehydrated in-gel for 15 hours at $20^{\circ} \mathrm{C}$ using the IPGphore system (Amersham Biosciences, GE Healthcare Life Sciences, USA). IEF was carried out for a total of $80 \mathrm{kVh}$. The IPG gel strips were equilibrated with $6 \mathrm{M}$ urea, $75 \mathrm{mM}$ Tris- $\mathrm{HCl} \mathrm{pH} 8.8,29.3 \%$ glycerol, $2 \%$ SDS and $1 \%$ DTT for 15 min and then incubated with the same buffer containing $2.5 \%$ iodoacetamide instead of DTT for $15 \mathrm{~min}$. 2-DGE was performed using a $12.5 \%$ gel. After electrophoresis, gels were stained with Coomassie Brilliant Blue R-250 for 3 hours followed by destaining with $10 \%$ methanol and $10 \%$ acetic acid for 3 hours. Gels were analyzed using Image Master Platinum 6.0 (GE Healthcare Life Sciences, USA). 
Mass spectrometry

For the identification of proteins by mass spectrometry (MS), spots were excised from the Coomassie-stained 2-D gels and subjected to in-gel digestion using chymotrypsin. The chymotryptic peptides were subsequently analyzed using nanoAcquity UPLC coupled to a Synapt G1 HDMS mass spectrometer (Waters, Milford, MA). MS spectra were searched against a database containing 63,245 Triticum protein sequences downloaded from the NCBI non-redundant database (www.ncbi.nlm.nih.gov) and 151,173 Triticum protein sequences downloaded from UniProt (www.uniprot. org). MS/MS analyses were conducted at the Korea Basic Science Institute, Seoul, Korea.

\section{Results}

The allelic compositions of LMW-GS in 32 Korean cultivars were previously determined by SDS-PAGE (Park et al. 2011, Shin et al. 2012). Three alleles (c, d, e) were reported at the Glu-A3 locus, four alleles (b, d, h, i) were found at the Glu-B3 locus, and three alleles $(\mathrm{a}, \mathrm{b}, \mathrm{c})$ were reported at the Glu-D3 locus in these cultivars.
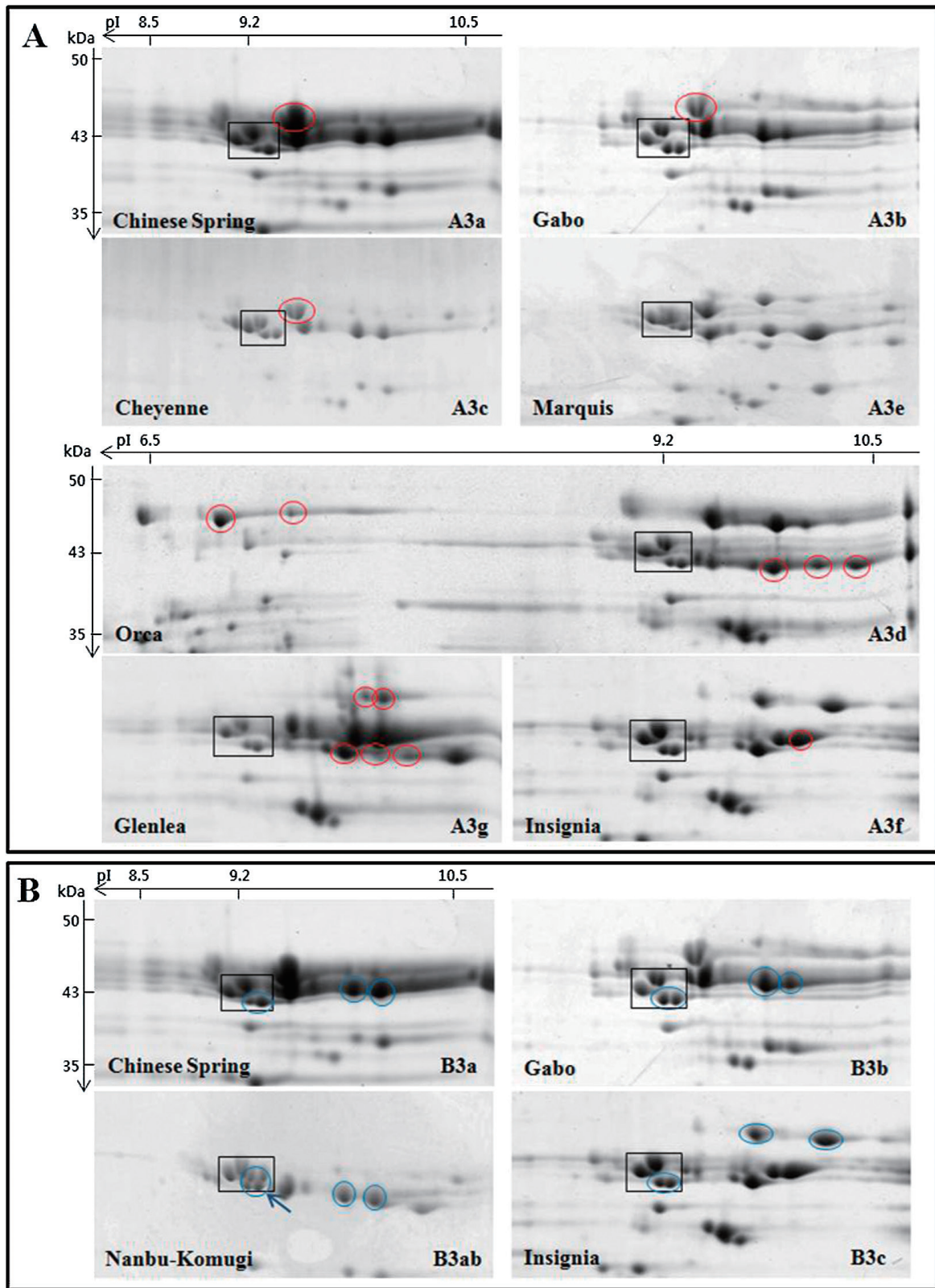

Fig. 1. LMW-GS profiles of standard wheat cultivars determined by 2-DGE. Only the regions of the gels containing LMW-GS are shown. Black boxes highlight LMW-GS proteins that are found in all cultivars and used as reference points among gels. Cultivar names and allelic designations are indicated on the lower left and right of each gel, respectively. A) Glu-A3 alleles. Characteristic proteins in each cultivar are shown in red circles. B) Glu-B3 alleles. Characteristic proteins in each cultivar are shown in blue circles. Blue arrows point to additional spots in the boxed areas. C) Glu-D3 alleles. Characteristic proteins in each cultivar are shown in green circles. 


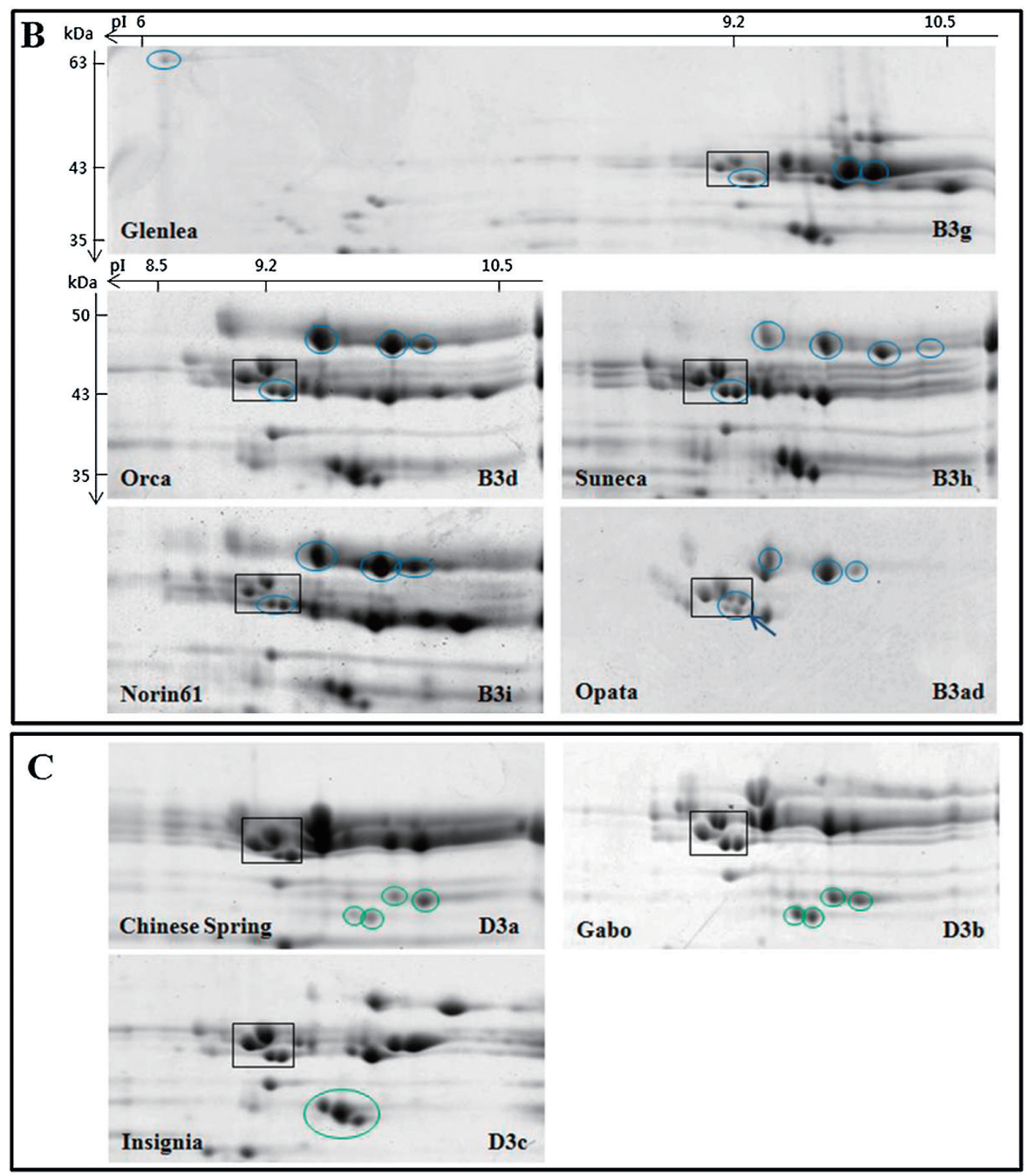

Fig. 1. (continued)

\section{2-DGE of standard cultivars}

LMW-GS profiles from the 32 cultivars were generated by 2-DGE and compared with profiles generated from a set of standard cultivars with known allelic compositions. Standard cultivars used for this analysis are shown in Table 1 and 2-DGE profiles of glutenin fractions from the cultivars are shown in Fig. 1. Interestingly, there are four spots with molecular weights of about $43 \mathrm{kDa}$ and isoelectric points of about 9.2 that are found in nearly all of the standard cultivars as well as most of the Korean cultivars. These spots are shown in black boxes in Fig. 1 and Fig. 2 and were used as reference points among the gels. Previous analysis of spots within the black box by tandem mass spectrometry in the Korean cultivar Keumkang demonstrated that all four spots correspond to m-type LMW-GS beginning with the N-terminal amino acid methionine. The two spots in the lower right corner of the box correspond to proteins encoded by the Glu-B3 locus while the two spots in the upper left portion of the box correspond to proteins encoded by the Glu-D3 locus (Lee et al. 2016).
These common LMW-GS spots were also reported by Ikeda et al. (2006). Only two Korean cultivars, Hanbaek and Uri, do not contain all four spots in this region (Fig. 2A, 2B, black arrows).

The cultivars Chinese Spring, Gabo, Cheyenne, Orca, Marquis, Insignia and Glenlea were used as standards for the Glu-A3 alleles a, b, c, d, e, f and g, respectively (Table 1). $G l u-A 3 e$ is a null allele. With the exception of Glu-A3a and $G l u-A 3 b$, the patterns of spots corresponding to the Glu-A3 alleles were readily distinguishable in the standard cultivars (Fig. 1A). The cultivars Chinese Spring, Gabo, Insignia, Orca, Glenlea, Suneca, Norin 61, Nanbu-Komugi, and Opata were used to represent the Glu-B3 alleles a, b, c, d, g, $\mathrm{h}, \mathrm{i}, \mathrm{ab}$ and ad, respectively (Table 1 ). With the exception of $G l u-B 3 d$ and $G l u-B 3 i$, all alleles could be distinguished by 2-DGE (Fig. 1B). The Glu-D3 alleles a, b and c were represented by the cultivars Chinese Spring, Gabo and Insignia, respectively (Table 1). Glu-D3a and Glu-D3b alleles are not readily distinguished by 2-DGE (Fig. 1C). 
Allelic analysis of Korean wheat cultivars at Glu-3 loci by 2-DGE

At the Glu-A3 locus, 13 of the 32 Korean cultivars contained 2-DGE spots characteristic of the Glu-A3c allele (Fig. 2A, red circles; Table 2). Twelve cultivars contained spots characteristic of the Glu-A3d allele (Fig. 2B, red circles; Table 2) and two cultivars (Jinpoom, Seodun) contained three spots that are characteristic of the Glu-A3d allele, but did not contain the two higher MW spots in the acidic portion of the gel (Fig. 2C, red circles). Since the 2-DGE profile does not match that from any of the seven standard Glu-A3 cultivars, Jimpoom and Seodum probably contain a new unidentified $G l u-A 3$ allele, referred to here as $U I$. Two cultivars, Jonong and Sinmichal 1 contained the five spots that were indicative of the Glu-A3g allele (Fig. 2D, red circles; Table 2). Three cultivars, Anabaek, Joeun and $\mathrm{Ol}$, contained the null allele, Glu-A3e, and did not have any spots corresponding to proteins encoded at the Glu-A3 locus (Fig. 2A, Table 2).

At the $G l u-B 3$ locus, 20 Korean cultivars contained the five spots characteristic of the Glu-B3d and Glu-B3i alleles (Fig. 2A, 2B, 2C, blue circles; Table 2). Four cultivars

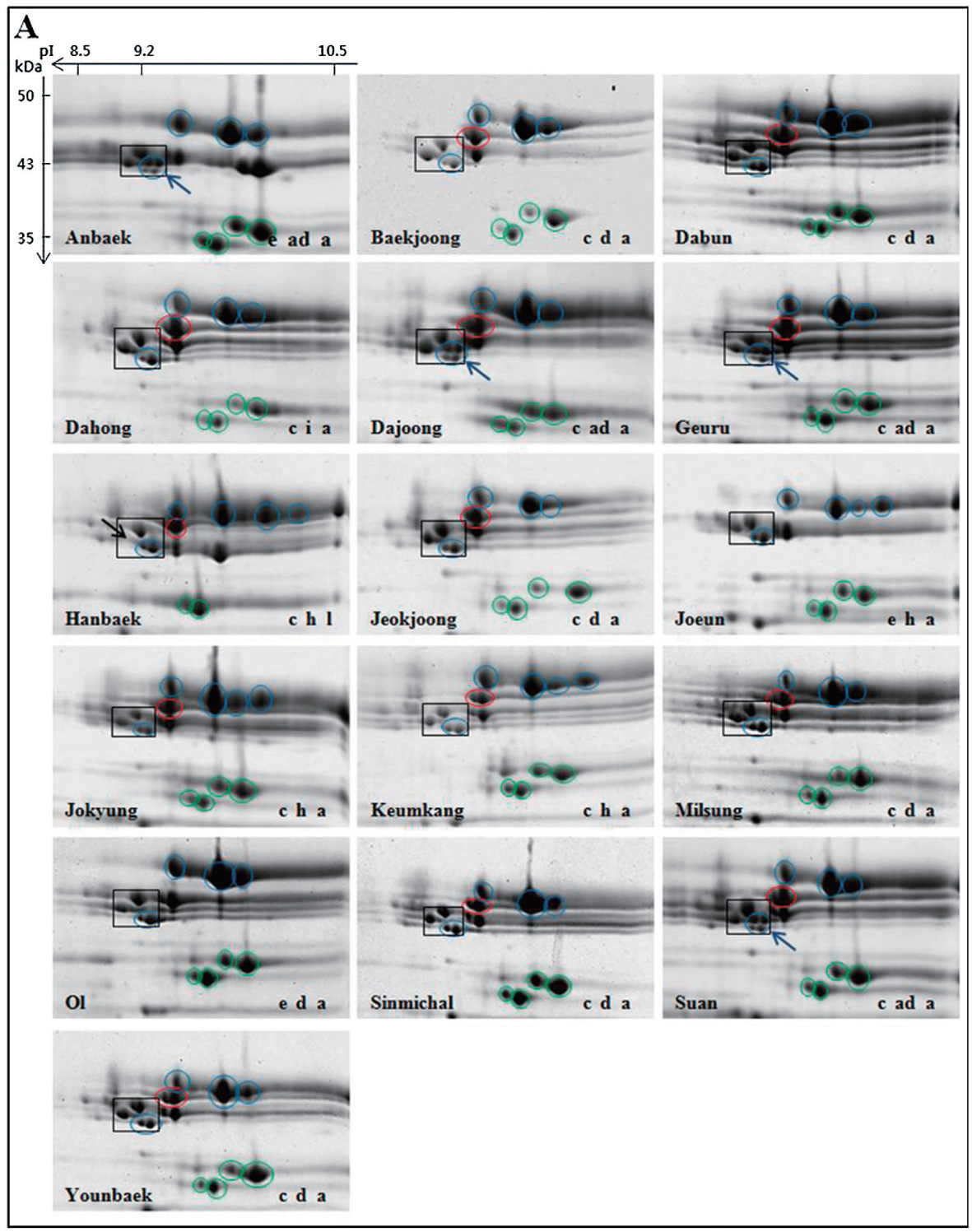

Fig. 2. LMW-GS profiles of Korean wheat cultivars determined by 2-DGE. Only the regions of the gels containing LMW-GS are shown. Black boxes highlight LMW-GS proteins that are found in most cultivars and used as reference points among gels. Spots corresponding to $G l u-A 3$, Glu-B3 and Glu-D3 alleles are shown in red, blue and green circles, respectively. Black arrows point to spots that are missing in the boxed areas. Blue arrows point to additional spots in the boxed areas. Cultivars are arranged in alphabetical order from left to right within each panel. Cultivar names and allelic designations are indicated on the lower left and right of each gel, respectively. Panels B and D include spots located in the acidic portion of the gels that are characteristic of the Glu-A3d and Glu-B3g alleles, respectively while Panel C demonstrates that cultivars Jinpoom and Seodun do not contain spots in the acidic portion of the gel that are characteristic of the Glu-A3d allele. 

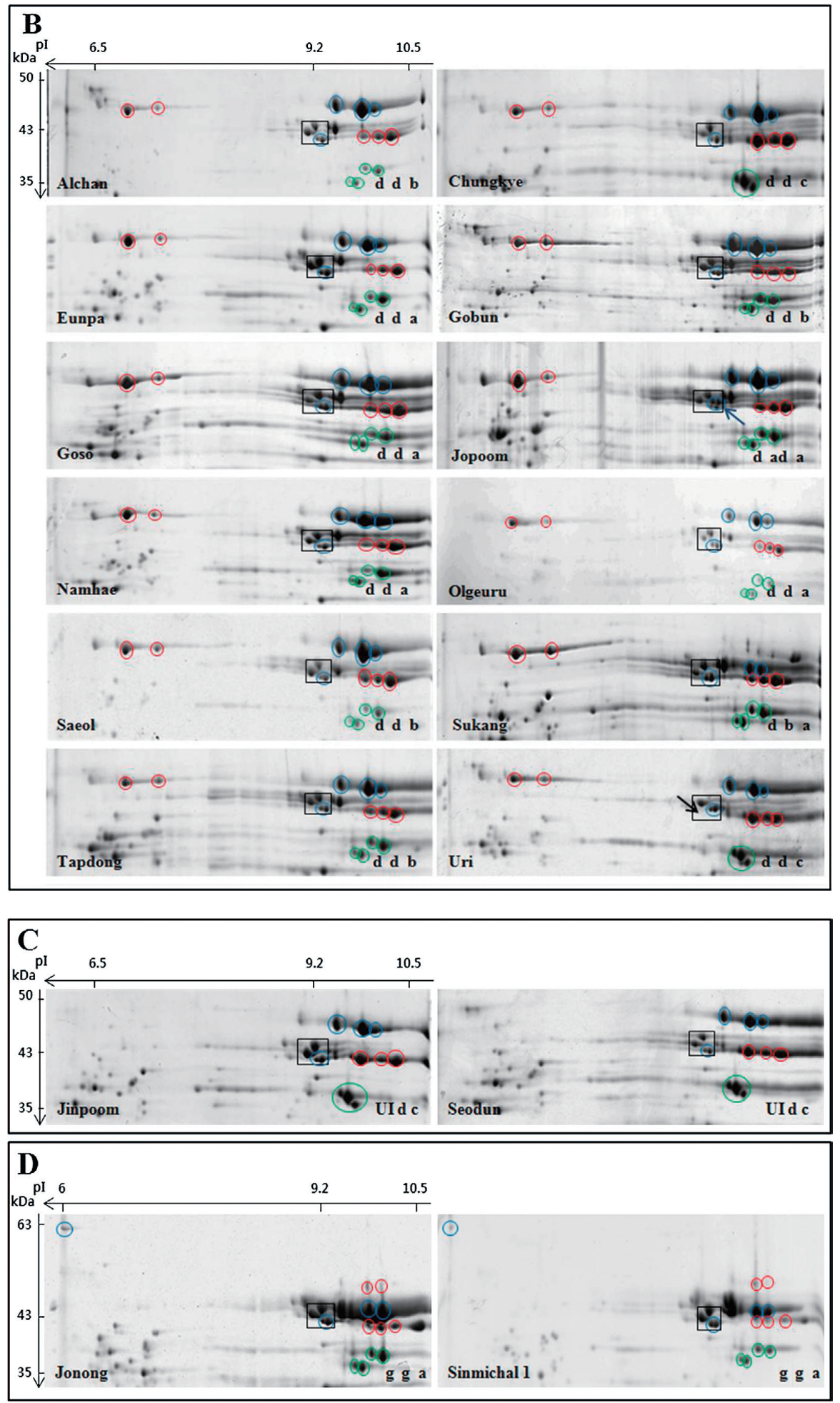

Fig. 2. (continued)

contained the six spots characteristic of the Glu-B3h allele (Fig. 2A, blue circles; Table 2). Only one cultivar, Sukang, contained the four spots typical of the $G l u-B 3 b$ allele (Fig. 2B, blue circles; Table 2). Two cultivars (Jonong, Sinmichal 1) contained the same Glu-B3 spots as Sukang, but also contained the higher MW protein on the acidic front of the gel that is characteristic of the Glu-B3g allele (Fig. 2D, blue circles; Table 2). Finally, five cultivars (Anbaek, Dajoong, Geuru, Suan and Jopoom) had 2-DGE profiles that were similar to those with the Glu-B3d and 
Table 2. Allelic compositions of LMW-GS at the Glu-A3, Glu-B3 and Glu-D3 loci in Korean cultivars determined by SDS-PAGE and 2-DGE. Cultivars are listed in alphabetical order along with the pedigree for each cultivar

\begin{tabular}{|c|c|c|c|c|c|c|c|c|c|}
\hline \multirow{3}{*}{ Cultivar } & \multirow{3}{*}{ Pedigree } & \multicolumn{6}{|c|}{ LMW-GS alleles } & \multirow{3}{*}{$\begin{array}{l}\text { Reference } \\
\text { Figure }\end{array}$} & \multirow{3}{*}{$\begin{array}{c}\text { Breeding } \\
\text { years }\end{array}$} \\
\hline & & \multicolumn{3}{|c|}{ SDS-PAGE $^{a}$} & \multicolumn{3}{|c|}{$2-\mathrm{DGE}^{b}$} & & \\
\hline & & Glu-A3 & Glu-B3 & Glu-D3 & Glu-A3 & Glu-B3 & Glu-D3 & & \\
\hline Alchan & Suwon210/Tapdong & $\mathrm{d}$ & $\mathrm{d}$ & $\mathrm{b}$ & $\mathrm{d}$ & $\mathrm{d}^{c}$ & $\mathrm{~b}^{d}$ & $2 \mathrm{~B}$ & 1994 \\
\hline Anbaek & Sae/Geuru & $\mathrm{e}$ & $\mathrm{i}$ & a & $\mathrm{e}$ & $\underline{\text { ad }}$ & $\mathrm{a}^{d}$ & $2 \mathrm{~A}$ & 2001 \\
\hline Baekjoong & Keumkang/Olgeuru & $\mathrm{c}$ & d & a & c & $\overline{\mathrm{d}^{c}}$ & $\mathrm{a}^{d}$ & $2 \mathrm{~A}$ & 2007 \\
\hline Chungkye & Norin4/Sharbatisonora & d & d & $\mathrm{c}$ & d & $\mathrm{d}^{c}$ & $\mathrm{c}$ & $2 \mathrm{~B}$ & 1979 \\
\hline Dabun & Suwon234//sw76039/Suwon220/3/Keumkang & $\mathrm{c}$ & d & a & $\mathrm{c}$ & $\mathrm{d}^{c}$ & $\mathrm{a}^{d}$ & $2 \mathrm{~A}$ & 2006 \\
\hline Dahong & Norin72/Wonkwang & $\mathrm{c}$ & $\mathrm{i}$ & $\mathrm{a}$ & c & $i^{c}$ & $\mathrm{a}^{d}$ & $2 \mathrm{~A}$ & 1979 \\
\hline Dajoong & SW992114-NM-131-7/Gobun & $\mathrm{c}$ & $\mathrm{i}$ & $\mathrm{a}$ & c & $\underline{\text { ad }}$ & $\mathrm{a}^{d}$ & $2 \mathrm{~A}$ & 2010 \\
\hline Eunpa & Chugoku81/3/Tob-CNO// Yuksung3/Suwon 185 & $\mathrm{~d}$ & $\mathrm{~d}$ & $\mathrm{a}$ & d & $\frac{\mathrm{du}^{c}}{\mathrm{~d}^{c}}$ & $\mathrm{a}^{d}$ & $2 \mathrm{~B}$ & 1982 \\
\hline Geuru & Strampelli/69D-3607//Chokwang & $\mathrm{c}$ & $\mathrm{i}$ & $\mathrm{a}$ & $\mathrm{c}$ & ad & $\mathrm{a}^{d}$ & $2 \mathrm{~A}$ & 1980 \\
\hline Gobun & Eunpa/Tapdong//Eunpa/Shannung6521 & $\mathrm{d}$ & $\mathrm{d}$ & $\mathrm{b}$ & $\mathrm{d}$ & $\frac{\mathrm{d}^{c}}{4}$ & $\mathrm{~b}^{d}$ & $2 \mathrm{~B}$ & 1995 \\
\hline Goso & Gobun/Ol & $\mathrm{d}$ & $\mathrm{d}$ & $\mathrm{a}$ & $\mathrm{d}$ & $\mathrm{d}^{c}$ & $\mathrm{a}^{d}$ & $2 \mathrm{~B}$ & 2010 \\
\hline Hanbaek & Shann7859/Keumkang//Guamuehill & $\mathrm{c}$ & $\mathrm{h}$ & $\mathrm{c}$ & $\mathrm{c}$ & $\mathrm{h}$ & $\underline{1}$ & $2 \mathrm{~A}$ & 2008 \\
\hline Jeokjoong & Keumkang/Tapdong & $\mathrm{c}$ & $\mathrm{d}$ & $\mathrm{a}$ & c & $\mathrm{d}^{c}$ & $\mathrm{a}^{d}$ & $2 \mathrm{~A}$ & 2007 \\
\hline Jinpoom & Geuru/Genaro81 & $\mathrm{d}$ & d & $\mathrm{c}$ & $\underline{\mathbf{U I}}^{e}$ & $\mathrm{~d}^{c}$ & $\mathrm{c}$ & $2 \mathrm{~A}$ & 1998 \\
\hline Joeun & Eunpa/Suwon242 & $\mathrm{e}$ & $\mathrm{h}$ & a & $\mathrm{e}$ & $\mathrm{h}$ & $\mathrm{a}^{d}$ & $2 \mathrm{~A}$ & 2000 \\
\hline Jokyung & Seri82/Keumkang & $\mathrm{c}$ & $\mathrm{h}$ & a & $\mathrm{c}$ & $\mathrm{h}$ & $\mathrm{a}^{d}$ & $2 \mathrm{~A}$ & 2004 \\
\hline Jonong & Suwon234/SW80199 & $\mathrm{c}$ & $\mathrm{h}$ & a & g & g & $\mathrm{a}^{d}$ & $2 \mathrm{C}$ & 2003 \\
\hline Jopoom & Kanto75//OR8500494P/Bezostaya & $\mathrm{d}$ & $\mathrm{i}$ & $\mathrm{a}$ & $\frac{\mathrm{s}}{\mathrm{d}}$ & $\underline{\underline{a}} \mathbf{a}$ & $\mathrm{a}^{d}$ & $2 \mathrm{~B}$ & 2001 \\
\hline Keumkang & Geuru/Kanto75//Eunpa & $\mathrm{c}$ & $\mathrm{h}$ & a & $\mathrm{c}$ & $\mathrm{h}$ & $\mathrm{a}^{d}$ & $2 \mathrm{~A}$ & 1996 \\
\hline Milsung & Shirogane//Norin43/Sonalika & $\mathrm{c}$ & $\mathrm{d}$ & a & $\mathrm{c}$ & $\mathrm{d}^{c}$ & $\mathrm{a}^{d}$ & $2 \mathrm{~A}$ & 1998 \\
\hline Namhae & Ol/Calidad & $\mathrm{d}$ & $\mathrm{d}$ & $\mathrm{a}$ & $\mathrm{d}$ & $\mathrm{d}^{c}$ & $\mathrm{a}^{d}$ & $2 \mathrm{~B}$ & 1988 \\
\hline $\mathrm{Ol}$ & Norin $72 /$ Norin 12 & $\mathrm{e}$ & $\mathrm{d}$ & $\mathrm{a}$ & $\mathrm{e}$ & $\mathrm{d}^{c}$ & $\mathrm{a}^{d}$ & $2 \mathrm{~A}$ & 1976 \\
\hline Olgeuru & Geuru/Chokwang//Saikai143 & $\mathrm{d}$ & $\mathrm{d}$ & a & $\mathrm{d}$ & $\mathrm{d}^{c}$ & $\mathrm{a}^{d}$ & $2 \mathrm{~B}$ & 1993 \\
\hline Saeol & Shirogane//Norin $43 /$ Sonalika & d & $\mathrm{d}$ & $\mathrm{b}$ & d & $\mathrm{d}^{c}$ & $\mathrm{~b}^{d}$ & $2 \mathrm{~B}$ & 1997 \\
\hline Seodun & Geuru/Genaro 81 & d & $\mathrm{d}$ & $\mathrm{c}$ & $\underline{\mathbf{U I}}^{e}$ & $\mathrm{~d}^{c}$ & $\mathrm{c}$ & $2 \mathrm{~A}$ & 1997 \\
\hline Sinmichal & Olgeuru//Kanto107/BaiHuo & $\mathrm{c}$ & d & $\mathrm{a}$ & $\mathrm{c}$ & $\mathrm{d}^{c}$ & $\mathrm{a}^{d}$ & $2 \mathrm{~A}$ & 2002 \\
\hline Sinmichall & Alchan//Kanto107/BaiHuo & $\mathrm{c}$ & b & a & g & g & $\mathrm{a}^{d}$ & $2 \mathrm{C}$ & 2006 \\
\hline Suan & Keumkang/Eunpa//Keumkang & $\mathrm{c}$ & $\mathrm{i}$ & a & $\frac{\mathrm{c}}{\mathrm{c}}$ & $\underline{\underline{a} d}$ & $\mathrm{a}^{d}$ & $2 \mathrm{~A}$ & 2009 \\
\hline Sukang & Suwon266/Asakaze & $\mathrm{d}$ & $\mathrm{b}$ & a & $\mathrm{d}$ & $\bar{b}$ & $\mathrm{a}^{d}$ & $2 \mathrm{~B}$ & 2008 \\
\hline Tapdong & Chugoku81//Suwon158/Toropi & $\mathrm{d}$ & $\mathrm{d}$ & $\mathrm{b}$ & $\mathrm{d}$ & $\mathrm{d}^{c}$ & $\mathrm{~b}^{d}$ & $2 \mathrm{~B}$ & 1986 \\
\hline Uri & Geuru/Ol & $\mathrm{d}$ & $\mathrm{d}$ & $\mathrm{c}$ & $\mathrm{d}$ & $\mathrm{d}^{c}$ & $\mathrm{c}$ & $2 \mathrm{~B}$ & 1992 \\
\hline Younbaek & Keumkang/Tapdong & $\mathrm{c}$ & $\mathrm{d}$ & $\mathrm{a}$ & c & $\mathrm{d}^{c}$ & $\mathrm{a}^{d}$ & $2 \mathrm{~A}$ & 2005 \\
\hline
\end{tabular}

${ }^{a}$ Allelic determinations from Park et al. (2011) and Shin et al. (2012).

${ }^{b}$ Allelic designations determined by 2-DGE that differ from those determined by SDS-PAGE are indicated in underlined boldface type.

${ }^{c}$ Glu-B3d and Glu-B3i alleles were not readily distinguished by 2-DGE, but can be distinguished by PCR (Wang et al. 2009).

${ }^{d} G l u-D 3 a$ and $G l u-D 3 b$ alleles were not readily distinguished by 2-DGE, but can be distinguished by SDS-PAGE.

${ }^{e}$ denotes new unidentified allele.

$G l u-B 3 i$ alleles, but contained two additional minor spots in the lower right corner of the black box (indicated by blue arrows in Fig. 2A, 2B). These profiles were similar to standard cultivar Opata containing Glu-B3ad.

Because the spot that distinguishes the Glu-B3b allele in the standard cultivar Gabo and the Korean cultivar Sukang from the $G l u-B 3 g$ allele found in the standard cultivar Glenlea and the Korean cultivars Jonong and Sinmichal 1 migrates at the acidic front of the gel, 2-DGE was conducted using a pI range of 3-11 instead of 6-11 (Fig. 3). A single spot of $\sim 63 \mathrm{kDa}$ was observed between the HMW-GS and gliadins in the acidic region of the gel in Glenlea, Jonong and Sinimichal 1, but not in Gabo or Sukang. The $63 \mathrm{kDa}$ spot in Glenlea was excised from the gel, digested with chymotrypsin, analyzed by tandem mass spectrometry and identified as a D-type LMW-GS (UniProtKB Accession B6ETS0) on the basis of the peptides SQQQISQQPQQ LPQQQQIPQQPQQF, QQYPQQQPSGSDVISICGL, and PQQPQQF. D-type LMW-GS have sequences like omega gliadins but contain a single cysteine residue and are encoded at the closely linked Gli-B1 locus.

At the Glu-D3 locus, 27 of the Korean cultivars contained the four spots that are characteristic of the Glu-D3a and $G l u-D 3 b$ alleles. Because these two alleles could not be distinguished by 2-DGE, the allelic designations determined by SDS-PAGE were considered to be confirmed. As shown in Fig. 4, these alleles can be distinguished by SDS-PAGE. Four cultivars contained the three spots characteristic of the Glu-D3c allele (Jinpoom, Seodun, Chungkye, Uri) (Fig. 2B, 2C, green circles; Table 2). The 2-DGE profile of one cultivar, Hanbaek, did not correspond to any of the Glu-D3 alleles shown in Fig. 1. This cultivar contained two spots that are consistent with the Glu-D3l allele described in the cultivar Amadina by Liu et al. (2010). However, Liu et al. (2010) suggested that a third spot with a lower MW and more acidic pI was also characteristic of this allele. While Hanbaek contains a spot in a similar position, the protein in the spot has an $\mathrm{N}$-terminal sequence corresponding to a gamma gliadin 

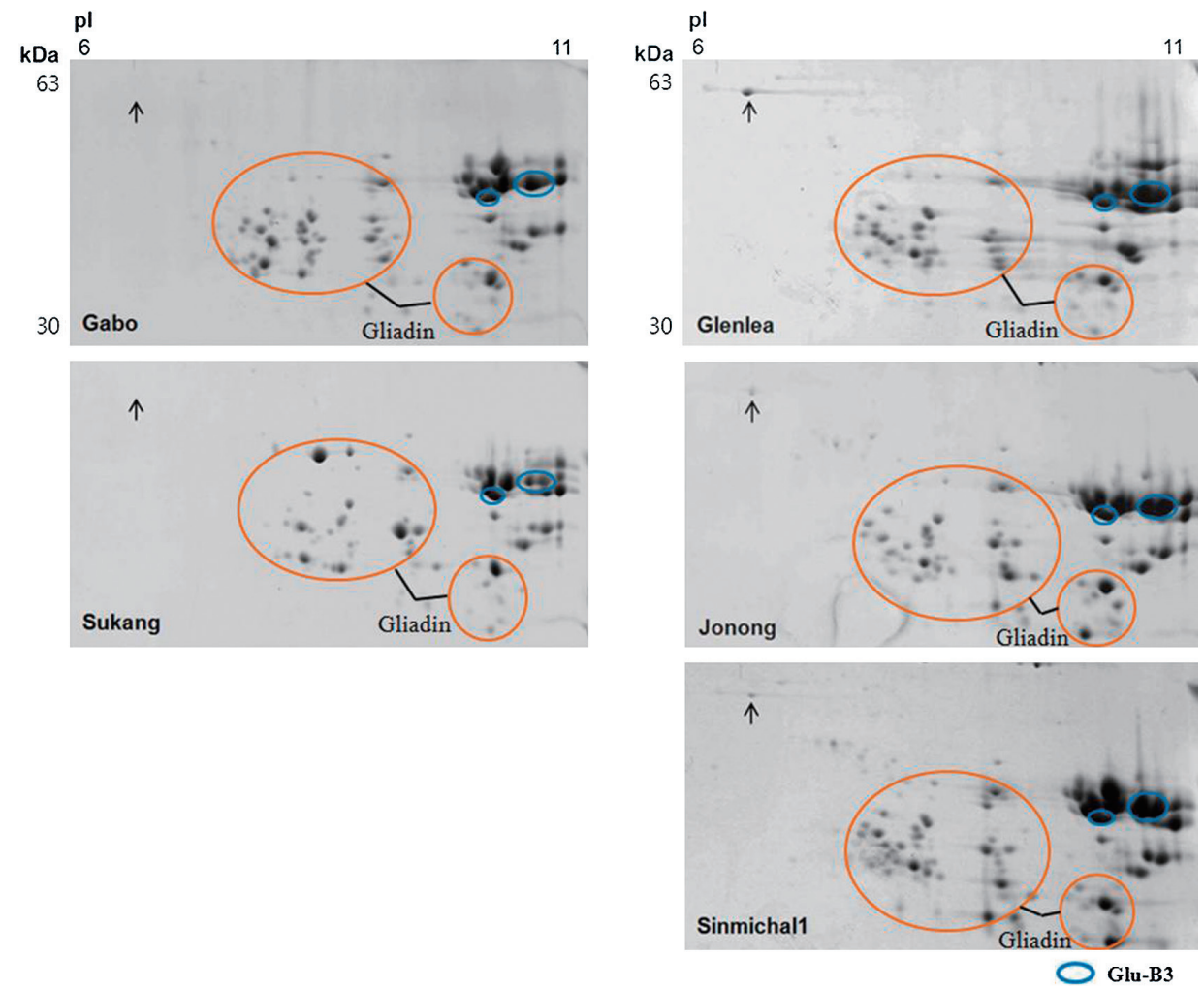

Fig. 3. 2-DGE patterns of glutenin fractions of standard wheat cultivars Gabo (Glu-B3b) and Glenlea (Glu-B3g) and Korean wheat cultivars Sukang, Jonong and Sinmichal 1 using a pI range of 3-11. Protein spots corresponding to Glu-B3 are shown in blue circles. Gliadins are indicated with orange circles. The position of the protein spot that distinguishes the $G l u-B 3 g$ allele from the $G l u-B 3 b$ allele is indicated with an arrow.

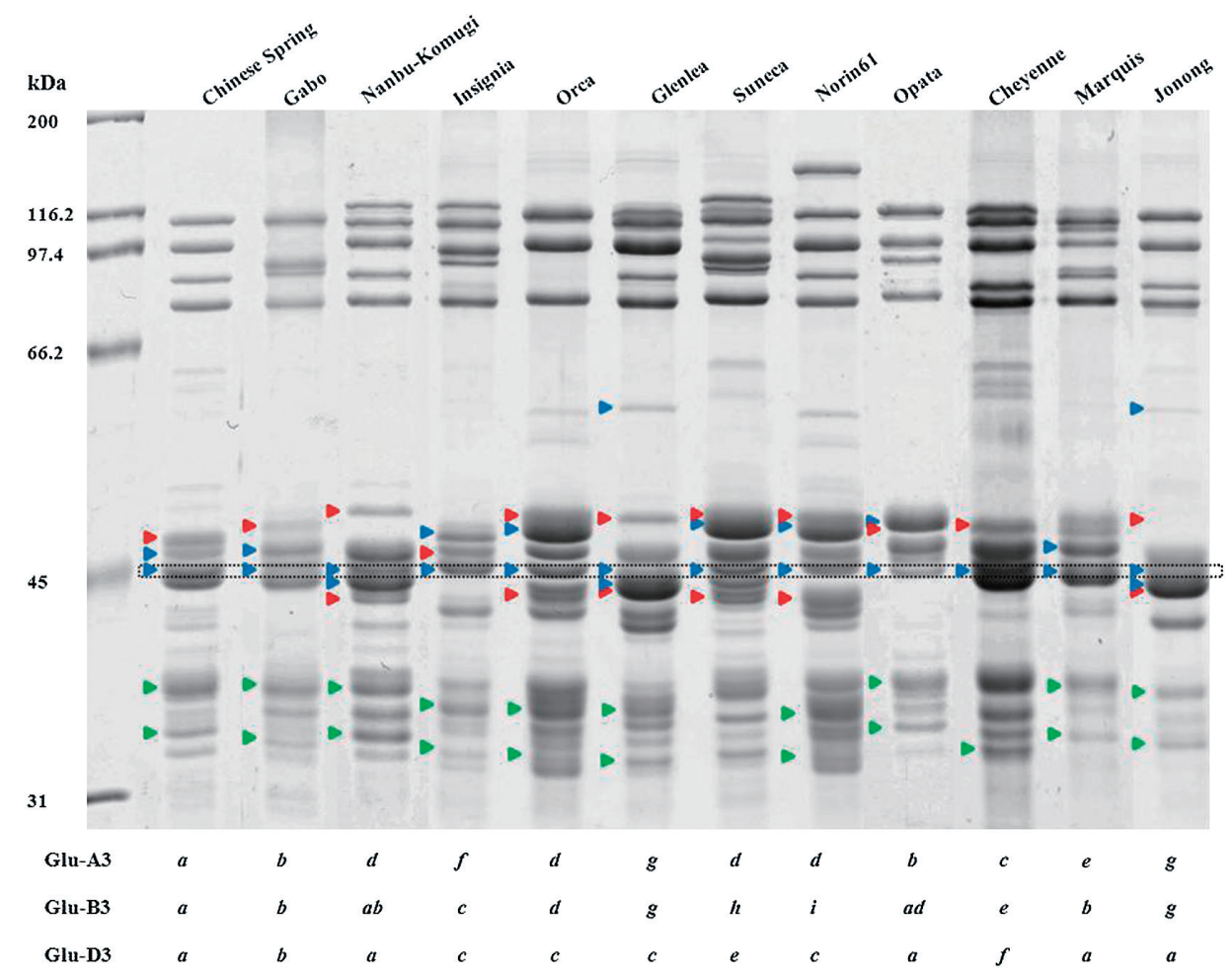

Fig. 4. Comparison of profiles of standard cultivars and Korean cultivar Jonong by SDS-PAGE. Red, blue and green arrows indicate the positions of bands characteristic of $G l u-A 3, G l u-B 3$ and $G l u-D 3$ alleles, respectively. Glu-B3 bands that are common to most cultivars are shown in the black dotted box. 
rather than a typical LMW-GS (data not shown).

There were discrepancies between the LMW-GS allelic designations determined by SDS-PAGE and by 2-DGE for ten of the 32 Korean cultivars (31\%) (Table 2). Four alleles at the Glu-A3 locus were scored incorrectly (13\%). In two cultivars, the $G l u-A 3 c$ allele should have been scored as the $G l u-A 3 g$ allele. In another two cultivars, the $G l u-A 3 d$ allele should have been scored as a new unidentified allele. Seven cultivars were scored incorrectly at the Glu-B3 locus by SDS-PAGE $(30 \%)$. In five cultivars, 2-DGE revealed that the Glu-B3i allele should have been scored as the Glu-3ad allele. In two other cases, either the Glu-B3h or the Glu-B3b allele should have been scored as a $G l u-B 3 g$ allele. Because the $G l u-B 3 h$ and $G l u-B 3 g$ alleles should be readily distinguished by SDS-PAGE, a separate sample from Jonong was analyzed by SDS-PAGE along with standard cultivars for all of the Glu-B3 alleles (Fig. 4). This analysis demonstrated that the Glu-B3 allele reported by Park et al. (2011) and Shin et al. (2012) in Jonong was incorrect and that the correct designation was $G l u-B 3 g$. Since the SDS-PAGE analysis of LMW-GS in Jonong was not shown in either report, it is impossible to determine whether the discrepancy was due to misidentification of gel bands or contamination of seed. Only one allele at the Glu-D3 locus was scored incorrectly. 2-DGE revealed that a Glu-D3c allele should have been scored as a $G l u-D 3 l$ allele.

\section{Discussion}

2-DGE was used to provide accurate information about the allelic compositions of LMW-GS in 32 Korean wheat cultivars. The study revealed five additional Glu-3 alleles not detected previously in these cultivars by SDS-PAGE. These included two alleles at the Glu-A3 locus (g and an unidentified allele, UI), two at the Glu-B3 locus (g, ad) and one at the Glu-D3 locus (1). The correct identification of the Glu$B 3$ alleles is important for breeding efforts to improve wheat quality since certain alleles are associated with favorable characteristics. Previous studies using near isogenic lines (NILs) demonstrated that Glu-A3d, Glu-B3b, Glu-B3g, and $G l u-B 3 i$ alleles are associated with superior bread-making quality, while Glu-A3e and $G l u-B 3 c$ alleles are related to inferior properties. Glu-D3 alleles made only slight differences in quality (Zhang et al. 2012). The Glu-A3d allele was found in 12 cultivars while the Glu-B3b was present in one, the $G l u-B 3 g$ in two, and Glu-B3i in one of the 32 Korean cultivars. Three cultivars contained the inferior Glu-A3e allele.

In this study, $31 \%$ of the cultivars were scored incorrectly using SDS-PAGE. It is noteworthy that a number of the alleles associated with good quality were misidentified by SDS-PAGE. Seven cultivars were incorrectly scored by SDS-PAGE as having good quality alleles. Two cultivars scored as $G l u-A 3 d$ by SDS-PAGE (Jinpoom, Seodun) had new unidentified alleles by 2-DGE and five cultivars scored as Glu-B3i by SDS-PAGE (Anbaek, Dajoong, Geuru, Jopoom, Suan) had Glu-B3ad alleles by 2-DGE. Additionally,
Table 3. Frequency of alleles for LMW-GS in 32 Korean wheat cultivars

\begin{tabular}{lccc}
\hline \hline Locus & Allele & Number of cultivars & Frequency $(\%)$ \\
\hline Glu-A3 & $c$ & 13 & 40.6 \\
& $d$ & 12 & 37.5 \\
& $e$ & 3 & 9.3 \\
& $g$ & 2 & 6.3 \\
& $U I$ & 2 & 6.3 \\
\hline Glu-B3 & $b$ & 1 & 3.1 \\
& $d$ & 19 & 59.4 \\
& $g$ & 2 & 6.3 \\
& $h$ & 4 & 12.5 \\
& $i$ & 5 & 3.1 \\
Glu-D3 & $a d$ & 23 & 15.6 \\
& $a$ & 4 & 71.9 \\
& $b$ & 4 & 12.5 \\
& $c$ & 1 & 12.5 \\
& $l$ & & 3.1 \\
\hline
\end{tabular}

one cultivar (Jonong) was scored incorrectly by SDS-PAGE as $G l u-B 3 h$, but was found to have the good quality allele Glu-B3g by 2-DGE. In this case, incorrect scoring of the LMW-GS allele may have been due to a mix-up in the seed sample.

Overall, the predominant LMW-GS alleles were the Glu$A 3 c$ and $G l u-A 3 d$, found in 41 and $38 \%$ of cultivars, respectively, the Glu-B3d found in 59\% and the Glu-D3a found in $72 \%$ of the cultivars (Table 3 ). The study suggests that Korean wheat cultivars have a very narrow genetic base with regards to the LMW-GS, probably because there was little selection for these genes in the past. Glu-A3c is also a predominant allele in wheat from the US, Australia and Argentina while the Glu-A3d allele is the predominant allele in wheat from China and also is prevalent in wheat from Japan and France (Branlard et al. 2003, Eagles et al. 2002, Lerner et al. 2009, Li et al. 2009, Shan et al. 2007, Tanaka et al. 2005). The Glu-B3d allele is moderately abundant in Chinese wheat, but rarely found in wheat from the US, Australia, Japan, France or Argentina. The most frequent $G l u-D 3$ allele in Korean cultivars, Glu-D3a, is also moderately abundant in US wheat.

Eight of the Korean cultivars analyzed in this study are frequently found in the pedigrees of other Korean cultivars (Alchan, Eunpa, Geuru, Gobun, Keumkang, Ol, Olgeuru, Tapdong). Of these, Alchan, Keumkang and Tapdong contain the Glu-Dld allele encoding HMW-GS subunits 1Dx5 and 1Dy10 that are associated with gluten strength. Alchan and Tapdong also contain the favorable $G l u-A 3 d$ allele. It is interesting that more than half of the cultivars released since 2000 contain Alchan, Keumkang or Tapdong in their backgrounds.

While the current work focused solely on Korean cultivars, it is likely that the lessons learned, particularly the high rate of misidentification of alleles resulting from SDSPAGE alone, also apply to cultivars being used in the breeding programs of other countries. The 2-DGE patterns generated in this study serve as reference maps of LMW-GS for 
most Korean cultivars that are not only important for wheat breeders but also will facilitate further study on the contributions of individual LMW-GS to flour functional properties. For example, it should be possible to analyze individual spots from these maps by tandem mass spectrometry and determine differences in the sequences of specific LMW-GS proteins that might relate to flour functional properties.

\section{Acknowledgements}

This work was supported by grants from the National Institute of Agricultural Science (RDA PJ012458), the RDA/ USDA-ARS Virtual Laboratory Program (RDA PJ012096), and the Next-Generation Bio-Green 21 Program (RDA PJ011021 and RDA PJ011057), Republic of Korea.

\section{Literature Cited}

Belton, P.S. (1999) Mini review: on the elasticity of wheat gluten. J. Cereal Sci. 29: 103-107.

Bradford, M. (1976) A rapid and sensitive method for the quantitation of microgram quantities of protein utilizing the principle of protein dye binding. Anal. Biochem. 72: 248-254.

Branlard, G., M.Dardevet, N.Amiour and G.Igrejas (2003) Allelic diversity of HMW and LMW glutenin subunits and omega-gliadins in French bread wheat (Triticum aestivum L.). Genet. Res. Crop Evol. 50: 669-679.

Eagles, H.A., G.J.Hollamby, N.N. Gororo and R.F.Eastwood (2002) Estimation and utilisation of glutenin gene effects from the analysis of unbalanced data from wheat breeding programs. Aust. J. Agric. Res. 53: 367-377.

Gupta, R.B. and K.W. Shepherd (1990) Two-step one-dimensional SDS-PAGE analysis of LMW subunits of glutelin. I. Variation and genetic control of the subunits in hexaploid wheats. Theor. Appl. Genet. 80: 65-74.

Gupta, R.B. and F. MacRitchie (1994) Allelic variation at glutenin subunit and gliadin loci, Glu-1, Glu-3 and Gli-1 of common wheats. II. Biochemical basis of the allelic effects on dough properties. J. Cereal Sci. 19: 19-29.

Ikeda, T.M., E.Araki, Y. Fujita and H. Yano (2006) Characterization of low-molecular-weight glutenin subunit genes and their protein products in common wheats. Theor. Appl. Genet. 112: 327-334.

Lee, J.Y., H.R.Beom, S.B.Altenbach, S.H.Lim, Y.T.Kim, C.S.Kang, U.H. Yoon, R. Gupta, S.T. Kim, S.N.Ahn et al. (2016) Comprehensive identification of LMW-GS genes and their protein products in a common wheat variety. Funct. Integr. Genomics 16: 269-279.

Lerner, S.E., M.A.Kolman and W.J.Rogers (2009) Quality and endosperm storage protein variation in Argentinean grown bread wheat. I. Allelic diversity and discrimination between cultivars. J. Cereal Sci. 49: 337-345.

Li, Y., C.Huang, X. Sui, Q. Fan, G. Li and X. Chu (2009) Genetic variation of wheat glutenin subunits between landraces and varieties and their contributions to wheat quality improvement in China. Euphytica 169: 159-168.

Liu, L., T.M.Ikeda, G. Branlard, R.J.Peña, W.J.Rogers, S.E.Lerner, M.A. Kolman, X. Xia, L. Wang, W. Ma et al. (2010) Comparison of low molecular weight glutenin subunits identified by SDS-PAGE, 2-DE, MALDI-TOF-MS and PCR in common wheat. BMC Plant Biol. 10: 124-148.
Luo, G., X.Zhang, Y.Zhang, W.Yang, Y.Li, J. Sun, K.Zhan, A.Zhang and D.Liu (2015) Composition, variation, expression and evolution of low-molecular-weight glutenin subunit genes in Triticum urartu. BMC Plant Biol. 15: 68.

Maruyama-Funatsuki,W., K. Takata, Z.Nishio, T.Tabiki, E. Yahata, A.Kato, K. Saito, H. Funatsuki, H. Saruyama and H. Yamauchi (2004) Identification of low-molecular weight glutenin subunits of wheat associated with bread-making quality. Plant Breed. 123: 355-360.

Park, C.S., C.S. Kang, J.U. Jeung and S.H. Woo (2011) Influence of allelic variations in glutenin on the quality of pan bread and white salted noodles made from Korean wheat cultivars. Euphytica 180: 235-250.

Payne, P.I., C.N. Law and E.E. Mudd (1980) Control by homoeologous group 1 chromosomes of the high-molecular-weight subunits of glutenin, a major protein of wheat endosperm. Theor. Appl. Genet. 58: $113-120$.

Payne, P.I. (1987) Genetics of wheat storage proteins and the effect of allelic variation on bread-making quality. Annu. Rev. Plant Physiol. 38: $141-153$.

Shan, X., S.R. Clayshulte, S.D. Haley and P.F. Byrne (2007) Variation for glutenin and waxy alleles in the US hard winter wheat germplasm. J. Cereal Sci. 45: 199-208.

Shewry, P.R., N.G. Halford and A.S. Tatham (1992) High molecular weight subunits of wheat glutenin. J. Cereal Sci. 15: 105-120.

Shin, S., C.S.Kang, J.U.Jeung, B.K.Baik, S.H. Woo and C.S. Park (2012) Influence of allelic variations of glutenin and puroindoline on flour composition, dough rheology and quality of white salted noodles from Korean wheat cultivars. Korean J. Breed. Sci. 44: 406-420.

Singh, N.K., K.W. Shepherd and G.B.Cornish (1991) A simplified SDS-PAGE procedure for separating LMW subunits of glutenin. J. Cereal Sci. 14: 203-208.

Tanaka,H., S. Toyoda and H.Tsujimoto (2005) Diversity of lowmolecular-weight glutenin subunit genes in Asian common wheat. Breed. Sci. 55: 349-354.

Wang, L., X.L.Zhao, Z.H.He, W.Ma, R.Appels, R.J.Peña and X.C.Xia (2009) Characterization of low-molecular-weight glutenin subunit Glu-B3 genes and development of STS markers in common wheat (Triticum aestivum L.). Theor. Appl. Genet. 118: $525-539$.

Wang, L.H., G.Y.Li, R.J.Peña, X.C. Xia and Z.H.He (2010) Development of STS markers and establishment of multiplex PCR for Glu-A3 alleles in common wheat (Triticum aestivum L.). J. Cereal Sci. 51: 305-312.

Zhang, W., M.C. Gianibelli, L.R. Rampling and K.R. Gale (2004) Characterisation and marker development for low molecular weight glutenin genes from Glu-A3 alleles of bread wheat (Triticum aestivum L). Theor. Appl. Genet. 108: 1409-1419.

Zhang, X., H.Jin, Y.Zhang, D. Liu, G.Li, X.Xia, Z.He and A.Zhang (2012) Composition and functional analysis of low-molecularweight glutenin alleles with Aroona near-isogenic lines of bread wheat. BMC Plant Biol. 12: 243.

Zhao, X.L., X.C.Xia, Z.H.He, K.R.Gale, Z.S.Lei, R.Appels and W.Ma (2006) Characterization of three low-molecular-weight Glu-D3 subunit genes in common wheat. Theor. Appl. Genet. 113: 1247-1259.

Zhen, S., C.Han, C.Ma, A. Gu, M.Zhang, X.Shen, X.Li and Y.Yan (2014) Deletion of the low-molecular-weight glutenin subunit allele Glu-A3a of wheat (Triticum aestivum L.) significantly reduces dough strength and breadmaking quality. BMC Plant Biol. 14: 367. 